\title{
Investigation of Carbapenemase Genes and Clonal Relationship in Carbapenem Resistant Klebsiella pneumoniae Strains
}

\author{
id Mustafa SAMASTI ${ }^{1}$, id Mücahide Esra KOÇOĞLU11, id İsmail DAVARCI², id Haluk VAHABOĞLU³ , id Hülya ÇAŞKURLU3 \\ I'stanbul Medeniyet University Faculty of Medicine, Department of Medical Microbiology, İstanbul, Turkey \\ ${ }^{2}$ Trakya University Faculty of Medicine, Department of Medical Microbiology, Edirne, Turkey \\ ${ }_{3}^{3}$ İstanbul Medeniyet University Faculty of Medicine, Department of Infectious Diseases, İstanbul, Turkey
}

\begin{abstract}
Objective: Resistant Gram-negative bacteria isolated from health-related infections are a worldwide problem. Increasing frequency of infections particularly caused by Enterobacteriaceae producing expanded spectrum beta lactamase, leads to the use of more carbapenem group antibiotics which, in turn, leads to bacterial resistance. In this study, we aimed to evaluate carbapenem resistance in Klebsiella pneumoniae (K. pneumoniae) isolates, the mechanisms causing this resistance and the clonal relationship between these isolates.

Methods: Ninety-one K. pneumoniae strains isolated from clinical samples obtained in our laboratory were included to the study. The identification of the bacteria was performed with Matriks assisted laser desorption ionization time of flight mass spectrometry (bioMérieux, Marcy-I'Étoile, France) and antimicrobial susceptibility with VITEK-2 (bioMérieux), and the carbapenem resistance was confirmed by ertapenem E-test (bioMérieux). Reverse transcription polymerase chain reaction method was used for the investigation of genes causing carbapenemase production $\left(b l a_{O X A-48}, b l a_{N D M-1}, b l a_{K P C}, b l a_{I M P}, b l a_{V I M-1}\right)$. The clonal relationship between isolates was investigated by pulsed-field jel elektroforez.

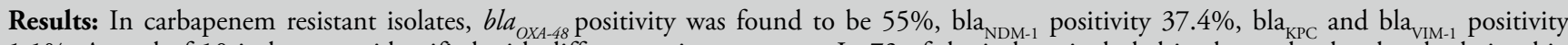
$1.1 \%$. A total of 10 isolates was identified with different resistance genes. In 73 of the isolates included in the study, the clonal relationship was examined, and 16 different groups were identified. Twenty isolates were not clonally associated with any other isolates. The most common resistance mechanism causing the carbapenem resistance was bla ${ }_{O X A-48}$ gene that is known to be endemic in Turkey.

Conclusion: As a result, the carbapenem resistance that we found as $3.13 \%$ in our study is similar to the rates obtained in other studies performed in our country which indicates that this resistance is not at a high level yet in our country. However, the ability of carbapenem resistance genes to spread between strains can be a major problem in the near future. Molecular methods are gold standard in carbapenemase detection, but because of having high cost they can not be used in laboratories routinely. Modified Hodge test or carbapenemase inactivation test are alternative tests with low costs that can be used in the determination of carbapenemase.
\end{abstract}

Keywords: Carbapenem, Klebsiella pneumoniae, resistance gene, pulsed-field gel electrophoresis

\section{Introduction}

Resistant Gram-negative bacteria isolated from health-related infections pose a worldwide problem (1). The incidence of infections is increasing in which bacteria, especially from the Enterobacteriaceae family, producing extended spectrum beta lactamase (ESBL), are isolated, leading to the further use of carbapenem group antibiotics (2).

Carbapenem resistance is mainly caused by two mechanisms: (i) overproduction of ESBL and/or AmpC along with the loss of porin, (ii) production of carbapenemase (3). Carbapenemases

Address for Correspondence: Mücahide Esra KOÇOĞLU, istanbul Medeniyet University Faculty of Medicine, Department of Medical Microbiology, İstanbul, Turkey

Phone: +90 21628031 56 E-mail: kocogluesra@yahoo.com ORCID ID: orcid.org/0000-0002-2860-1794

Cite this article as: Samastı M, Koçoğlu ME, Davarcı I, Vahaboğlu H, Çaşkurlu H. Investigation of Carbapenemase Genes and Clonal Relationship in Carbapenem Resistant Klebsiella pneumoniae Strains. Bezmialem Science 2019;7(3):186-90. 
are classified in class A (bla ${ }_{\mathrm{KPC}}$ vb.), B (bla ${ }_{\mathrm{VIM}-1}$, bla $\mathrm{IMP}_{\mathrm{IMP}}, \mathrm{bla}_{\mathrm{NDM}-1}$ vb.) and D (bla ${ }_{\text {OXA-48 }} v$ vb.) according to the Ambler classification $(3,4)$. Phenotypic determination of carbapenem-producing Enterobacteriaceae members is difficult because carbapenems' minimum inhibitory concentrations may be low. Therefore, the genotypic determination of carbapenemase producing genes is gold standard (5).

In this study, we aimed to evaluate the carbapenem resistance in Klebsiella pneumoniae ( $K$. pneumoniae)strains, the identification of resistance genes that cause this resistance, and the clonal relationship between resistant strains.

\section{Methods}

\section{Bacterial Strains}

Ninety one strains resistant to carbapenem among $2903 \mathrm{~K}$. pneumoniae strains isolated in our hospital microbiology laboratory between January 2015 and December 2016 were included in this study. The identification of bacteria was made with Matriks assisted laser desorption ionization time of flight mass spectrometry (bioMérieux, Marcy-I'Etoile, France). İstanbul Medeniyet University Göztepe Training and Research Hospital Clinical Research Ethics Committee decision no: 2016/0034, 09.02.201.

\section{Antimicrobial Susceptibility Testing}

Antimicrobial susceptibility test of isolated strains (colistin, ampicillin/sulbactam, piperacilin/tazobactam, ceftazidime, ceftriaxone, erapenem, meropenem, imipenem, ciprofloxacin, levofloxacin, gentamicin, amikacin and trimethoprim/ sulfamethoxazole) was done with the automated system of VITEK-2 (bioMérieux, Marcy-I'Étoile, France). The authentication of strains with decreased sensitivity or resistance to any of the carbapenems was performed with erythromycin E-test (bioMérieux). Border values were evaluated in accordance with the recommendations of European Committee on Antimicrobial Susceptibility Testing (EUCAST) (6). K. pneumoniae American Type Culture Collection (ATCC) 700603 was used as the quality control strain.

\section{Investigation of the Genes that Produce Carbapenemase}

DNA extraction was carried out using the Qiagen (QIA)mp DNA mini kit (Qiagen GmbH, Hilden, Germany) in 18-24-hour culture of bacteria in accordance with the recommendations of the manufacturer. The genes causing carbapenemase production (bla ${ }_{\text {OXA-48 }}$, bla $_{\mathrm{NDM}-1}$, bla $_{\mathrm{KPC}}$, bla $_{\mathrm{IMP}}$, bla $_{\mathrm{VIM}-1}$ ) were evaluated with real-time polymerase chain reaction (PCR) in the Rotor-Gene Q device (Qiagen, Germany). The presence of the genes evaluated in the study was investigated using commercially produced Microbial DNA qPCR Assay (REF 330025) (Qiagen, USA) kits. Data obtained from PCR results were analyzed by setting the green channel threshold to 0.02 absolute value.

\section{Pulsed-field Gel Electrophoresis (PFGE)}

The epidemiological relation of carbapenem resistant $K$. pneumoniae (KDKp) strains was investigated in the the
Molecular Microbiology Laboratory of Turkish Public Health Institution using pulsed-field gel electrophoresis (PFGE). Bionumerics program 7.1 version was used to evaluate the results. Clustering analysis was performed using "unwighted pair group method with mathematical averaging". The relationship between the strains was determined according to "dice" similarity coefficient. Tolerance was taken as $1.5 \%$ and optimization as $1 \%$ in the calculation of similarity coefficient. PFGE types were determined according to the DNA tailing patterns, in accordance with the criteria defined by Tenover et al. (7) and the strains with a similarity of $\geq 85 \%$ were defined as "related strains". Normalization was done by using $K$. pneumoniae ATCC 700603 standard strain.

\section{Results}

Ninety-one $(3.13 \%)$ of a total of 2903 K. pneumoniae strains isolated from various clinical samples in our laboratory were found to be resistant to at least one antimicrobial agent from carbapenem group.

Of carbapenem resistant strains, $49.5 \%$ were isolated from intensive care unit, $12.1 \%$ from internal medicine, $11 \%$ from general surgery, $8.8 \%$ from brain surgery and $8.8 \%$ from child health and disease services and $9.8 \%$ from other services (emergency service, infectious diseases, neurology and urology). Of resistant strains, $58.2 \%$ were isolated from blood, $19.8 \%$ from tracheal aspirate, $11 \%$ from deep tissue aspirate, $8.8 \%$ from urine and $2.2 \%$ from phlegm culture. All strains were resistant to ertapenem, $93.3 \%$ to meropenem, $91.1 \%$ to imipenem. In addition, all KDKp strains were resistant to piperacillin/ tazobactam and ampicillin/sulbactam, 95.6\% to ceftazidime and ceftriaxon, $86.7 \%$ to ciprofloxacin and levofloxacin, $82.2 \%$ to trimethoprim/sulfamethoxazole. Resistance rates to colistin, amikacin and gentamicin were shown as $9 \%, 42.2 \%$ and $62.2 \%$, respectively and they were relatively sensitive antibiotics.

All strains were tested for bla $a_{\text {XXA-48 }}, b a_{N D M-1}, b_{\text {KPC }}, b_{\text {IMP }}$ and bla $_{\mathrm{VIM}-1}$ resistance genes, and bla $\mathrm{OXA}-48(55 \%)$ and bla $\mathrm{NDM}_{1}$ $(37.4 \%)$ were the most frequently found resistance genes (Figure 1). Resistance genes were not detected in a total of 15 strains and two different resistance genes were found in 10 strains.

Seventy three of 91 KDKp strains included in the study were investigated in terms of clonal relations with PFGE due to the technical problems experienced in the reanimation of bacteria. In

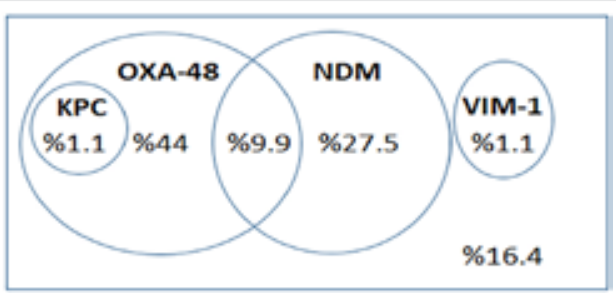

Figure 1. Distribution of resistance genes in carbapenem resistant 91 strains

KPC: Klebsiella pneumoniae carbapenemase, OXA: Oxacillin sodium, NDM: New Delhi metallo-beta-laktamaz-1, VIM: Vimentin 
20 of these strains, a clonal relationship was not detected, while others formed 16 clonal groups (Figure 2). The presence of $K$. pneumoniae strains carrying the same resistance genes in different groups suggested that mobile genetic elements carrying these resistance genes might be transferred horizontally.

\section{Discussion}

Carbapenem resistance has been spreading rapidly recently and is a major problem threatening public health. The solution of this problem requires studies to explain the mechanisms of resistance and the development of methods to determine the resistance to carbapenem quickly and easily $(3,8)$.

EUCAST indicates that ertapenem has high sensitivity and low specificity and meropenem has good sensitivity-specificity balance in detecting carbapenem resistance in Enterobacteriaceae (9). Therefore, in our study, ertapenem E-test was used to confirm carbapenem resistance. All strains were resistant to ertapenem and some strains were resistant to both meropenem and imipenem. Our findings supported that the use of ertapenem in detection of carbapenem resistance was more appropriate.

In the multi-center SENTRY study conducted in the United States (USA) between 2007-2009, carbapenem resistance in $K$.

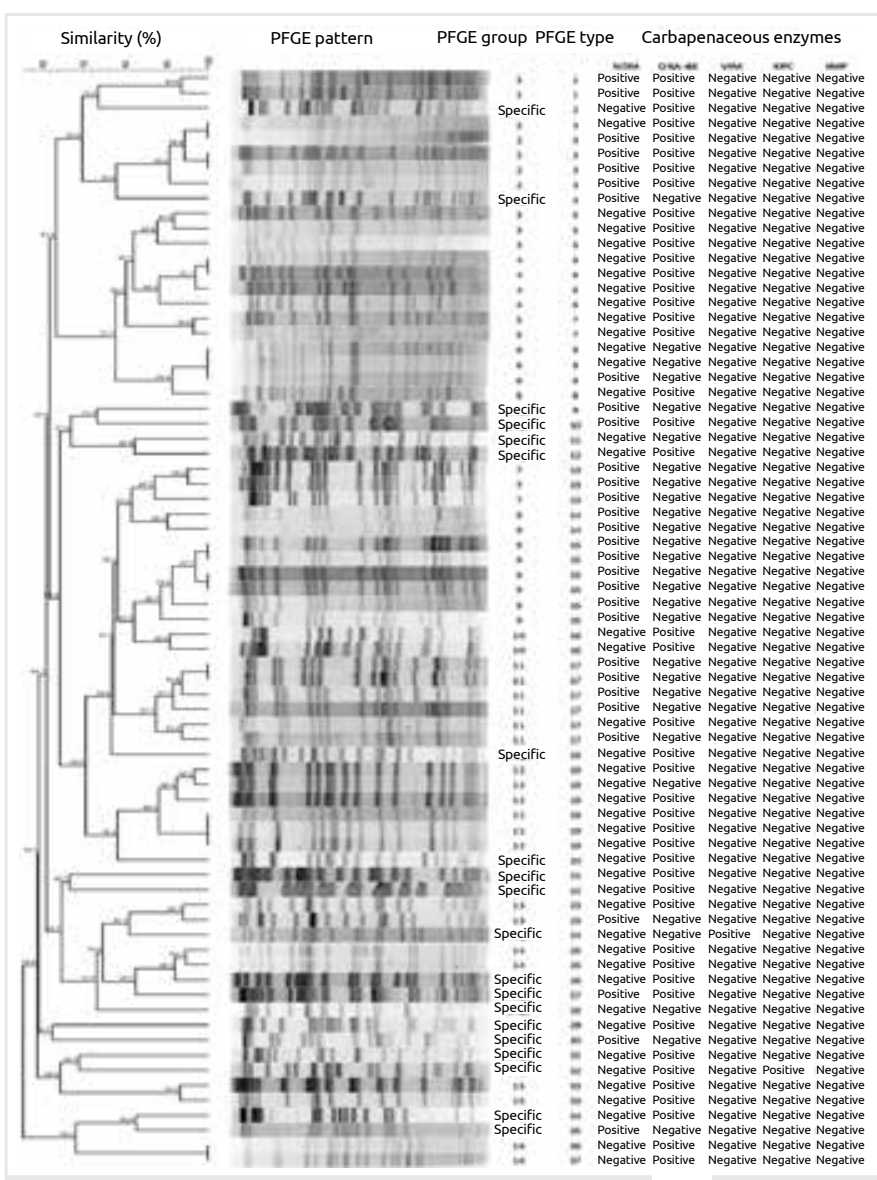

Figure 2. Clon analysis results of 73 strains by using pulsedfield gel electrophoresis

PFGE: Pulsed-field gel electrophoresis pneumoniae strains was found to be $6.1 \%$ (10). This resistance was $1-5 \%$ in the European

Antimicrobial Resistance Surveillance System-2008 study in Europe and $3.2 \%$ in the high intensity tactical training-2 study in Turkey $(11,12)$. Karbapenem resistance was found $3.13 \%$ in our study, consistent with the European and Turkish Data.

KDKp strains were most frequently isolated from intensive care units, compatible with another study in our country (3). Mechanical ventilation, prolonged intubation time, the use of paralytic agents and advanced age were found to increase the risk of infection. Even staying in the intensive care unit was stated as a risk factor alone (13).

All of the strains undergoing antimicrobial susceptibility test performed with KDKp strains were reported to be resistant to ertapenem. The most sensitive antimicrobial was reported as colistin $(3,14)$. The data we obtained in our study showed the same situation.

Carbapenemase resistance was first identified in Enterobacter clocae strains more than 20 years ago. During this time, a large number of carbapenem resistance has been reported in different bacteria with different resistance mechanisms from many countries of the world (15).

In the present study, we found that bla ${ }_{\mathrm{OXA}-48}$ and bla ${ }_{\mathrm{NDM}-1}$ were the most frequent carbapenem resistance genes $(55 \%$ and $37.4 \%$, respectively). $K$. pneumoniae strains carrying bla ${ }_{\text {OXA-48 }}$ are considered endemic in Turkey $(3,16,17)$. In a multi-center study conducted in Europe, $K$. pneumoniae strains with decreased sensitivity to carbapenem with bla ${ }_{\text {OXA-48 }}$ positivity was found to be $79 \%$ for Turkey (18). Tekintaş et al. (19) showed bla ${ }_{\text {OxA-48 }}$ positivity in 52 of $54(96.3 \%)$ KDKp strains in a study published in 2017 .

An endemic region for bla $\mathrm{NDM}_{\mathrm{N}-1}$ positive $K$. pneumoniae has not been identified It was seen in Sweden in 2015 and it was isolated from a patient in India in $2009(17,20)$. Bla ${ }_{\mathrm{NDM}-1}$ positivity in carbapenem resistant $K$. pneumoniae strains was detected in 2011 for the first time in Turkey in a patient with leukemia who underwent a bone marrow transplantation (3). In a multicenter study published in 2017, bla $_{\mathrm{NDM}-1}$ positivity was reported as $7.3 \%$ in KDKp strains in Turkey (18). Other studies in our country reported bla $\mathrm{NDM}-1$ positivity in $38.9 \%$ and $29.5 \%$ of strains $(19,21)$. These ratios coincide with our findings.

In our study, bla $a_{K P C}$ and $b l a_{V I M-1}$ genes were detected in one patient and bla $\mathrm{IMP}_{\mathrm{I}}$ gene was not detected. The presence of $\mathrm{bla}_{\mathrm{IMP}}$, bla $_{\mathrm{VIM}-1}$ and $\mathrm{bla}_{\mathrm{KPC}}$ in $K$. pneumoniae strains in Turkey was first detected in 2003, 2005 and 2014, respectively (22-24). In a study conducted in our country in 2016, only one of $279 \mathrm{KDKp}$ strains was found to have bla ${ }_{I M P}$ gene (25). These genes are endemic in some countries such as Italy and Greece $(15,17)$.

In our study, resistance genes other than the five most common carbapenemase enzymes in Turkey were not searched, which constituted the limitation of our study. PFGE patterns of a total of $73 \mathrm{KDKp}$ strains showed differences. Although isolating 
times of strains in group 1 were close to each other, the services they were isolated were different. Being isolated of the strains in groups $2,7,9,11$ and 15 at different times from the same service suggested that a health worker or material might be a reservoir. In other groups, it was observed that the strains were isolated in services at different times from each other. $K$. pneumoniae strains carrying the same resistance gene could be found in different groups or even they did not belong to any group, suggesting that mobile genetic elements carrying these resistance genes might be transferred horizontally between bacteria.

\section{Conclusion}

As a result, carbapenem resistance in our country is not yet at the highest level. This was found to be $3.13 \%$ in our study. However, the horizontal spread of the genes that cause carbapenem resistance can be a major problem in the near future. Because of the more deadly surveillance of infections with KDKp strains and the lack of treatment options; rapid and reliable tests are needed to determine resistance status (26-28). Although the molecular methods in detection of carbapenemase are gold standard, they can not be done routinely in each laboratory because of the high cost. Alternatively, practical and less costly methods such as carbapenemase inactivation test or modified hodge test can be used in the determination carbapenemase.

\section{Ethics}

Ethics Committee Approval: İstanbul Medeniyet University Göztepe Training and Research Hospital Clinical Research Ethics Committee decision no: 2016/0034, 09.02.201.

Informed Consent: Our study was an in vitro study.

Peer-review: Internally peer-reviewed.

\section{Authorship Contributions}

Concept: M.S., İ.D., M.E.K., Design: İ.D., M.E.K., Data Collection or Processing: İ.D., M.E.K., Analysis or Interpretation: İ.D., M.E.K., H.V., Literature Search: H.Ç., İ.D., M.E.K., Writing: İ.D., M.E.K.,

Conflict of Interest: No conflict of interest was declared by the authors.

Financial Disclosure: The authors declared that this study received no financial support.

\section{References}

1. Kollef MH, Fraser VJ. Antibiotic resistance in the intensive care unit. Ann Intern Med 2001;134:298-314.

2. Gülmez D, Woodford N, Palepou MF, Mushtaq S, Metan G, Yakupogullari Y et al. Carbapenem-resistant Escherichia coli and Klebsiella pneumoniae isolates from Turkey with OXA-48-like carbapenemases and outer membrane protein loss. Int J Antimicrob Agents 2008;31:523-6.

3. Baran I, Aksu N. Phenotypic and genotypic characteristics of carbapenem-resistant Enterobacteriaceaein a tertiary-level reference hospital in Turkey. Ann Clin Microbiol Antimicrob 2016;15:20.
4. Lascols C, Peirano G, Hackel M, Laupland KB, Pitout JD. Surveillance and Molecular Epidemiology ofKlebsiella pneumoniae Isolates That Produce Carbapenemases: First Report of OXA-48Like Enzymes in North America. Antimicrob Agents Chemother 2013;57:130-6.

5. Cohen Stuart J, Leverstein-Van Hall MA. Guideline for phenotypic screening and confirmation of carbapenemases in Enterobacteriaceae. Int J Antimicrob Agents 2010;36:205-10.

6. http://www.eucast.org/clinical_breakpoints/, EUCAST Breakpoint Tables v 7.1, Aralık 2017.

7. Tenover FC, Arbeit RD, Goering RV. How to select and interpret molecular strain typing methods for epidemiological studies of bacterial infections: a review for healthcare epidemiologists. Molecular Typing Working Group of the Society for Healthcare Epidemiology of America. Infect Control Hosp Epidemiol 1997;18:426-39.

8. Kuskucu MA, Karakullukcu A, Ailiken M, Otlu B, Mete B, Aygun $\mathrm{G}$. Investigation of carbapenem resistance and the first identification of Klebsiella pneumoniae carbapenemase (KPC) enzyme among Escherichia coli isolates in Turkey: A prospective study. Travel Med Infect Dis 2016;14:572-6.

9. http://www.eucast.org/resistance_mechanisms/ Temmuz 2017.

10. Kaiser RM, Castanheira M, Jones RN, Tenover F, Lynfield R. Trends in Klebsiella pneumoniae carbapenemase-positive K.pneumoniae in US hospitals: report from the 2007-2009 SENTRY Antimicrobial Surveillance Program. Diagn Microbiol Infect Dis 2013;76:356-60.

11. 11.https://ecdc.europa.eu/sites/portal/files/media/en/publications/ Publications/1011_SUR_annual_EARS_Net_2008.pdf

12. Gur D, Hascelik G, Aydin N, Telli M, Gültekin M, Ogunc D et al. Antimicrobial resistance in gram-negative hospital isolates: results of the Turkish HITIT-2 Surveillance Study of 2007. J Chemother 2009;21:383-9.

13. Rodríguez-Acelas AL, de Abreu Almeida M, Engelman B, CañonMontañez W. Risk factors for health care-associated infection in hospitalized adults: Systematic review and meta-analysis. Am J Infect Control 2017;45:e149-e156.

14. Lascols C, Peirano G, Hackel M, Laupland KB, Pitout JD. Surveillance and molecular epidemiology of Klebsiella pneumoniae isolates that produce carbapenemases: first report of OXA-48-like enzymes in North America. Antimicrob Agents Chemother 2013;57:130-6.

15. Nordmann P, Poirel L. The difficult-to-control spread of carbapenemase producers in Enterobacteriaceae worldwide. Clin Microbiol Infect 2014;20:821-30.

16. Poirel L, Héritier C, Tolün V, Nordmann P. Emergence of Oxacillinase-Mediated Resistance to Imipenem in Klebsiella pneumoniae. Antimicrob Agents Chemother 2004;48:15-22.

17. Albiger B, Glasner C, Struelens MJ, Grundmann H, Monnet DL ve group., European Survey of Carbapenemase-Producing Enterobacteriaceae (EuSCAPE) working. Carbapenemase-producing Enterobacteriaceae in Europe: assessment by national experts from 38 countries, May 2015. Euro Surveill 2015;20.

18. Grundmann H, Glasner C, Albiger B, Aanensen DM, Tomlinson CT, Andrasević AT et al. Occurrence of carbapenemase-producing Klebsiella pneumoniae and. Escherichia coli in the European survey 
of carbapenemase-producing Enterobacteriaceae (EuSCAPE): a prospective, multinational study. Lancet Infect Dis 2017;17:153-63.

19. Tekintaş Y, Çilli F, Eraç B, Yaşar M, Aydemir SŞ, Hoşgör Limoncu M. Comparison of phenotypic methods and polymerase chain reaction for the detection of carbapenemase production in clinical Klebsiella pneumoniae isolates. Mikrobiyol Bul 2017;51:269-76.

20. Rolain JM, Parola P, Cornaglia G. New Delhi metallo-beta-lactamase (NDM-1): towards a new pandemia? Clin Microbiol Infect 2010;16:1699-701.

21. Haciseyitoglu D, Dokutan A, Abulaila A, Erdem F, Cag Y, Ozer S et al. The First Enterobacter cloacae Co-Producing NDM and OXA-48 Carbapenemases and Interhospital Spread of OXA-48 and NDMProducing Klebsiella pneumoniae in Turkey. Clin Lab 2017;1:121322.

22. Labarca J, Poirel L, Ozdamar M, Turkoglü S, Hakko E, Nordmann P. KPC-producing Klebsiella pneumoniae, finally targeting Turkey. New Microbes New Infect 2014;2:50-1.

23. Yildirim I, Ceyhan M, Gur D, Mugnaioli C, Rossolini GM. First detection of VIM-1 type metallo-beta-lactamase in a multidrugresistant Klebsiella pneumoniae clinical isolate from Turkey also producing the CTX-M-15 extended-spectrum beta-lactamase. J Chemother 2007;19:467-8.

24. Aktaş Z, Poirel L, Salcioğlu M, Ozcan PE, Midilli K, Bal C et al. First IMP-1-producing Klebsiella pneumoniae isolate in Turkey. Clin Microbiol Infect 2006;12:695-6.

25. Zarakolu P, Eser OK, Aladag E, Al-Zahrani IA, Day KM, Atmaca O et al. Epidemiology of carbapenem-resistant Klebsiella pneumoniae colonization: a surveillance study at a Turkish university hospital from 2009 to 2013. Diagn Microbiol Infect Dis 2016;85:466-70.

26. Djahmi N, Dunyach-Remy C, Pantel A, Dekhil M, Sotto A, Lavigne JP. Epidemiology of Carbapenemase-Producing Enterobacteriaceae and Acinetobacter baumannii in Mediterranean Countries. Biomed Res Int 2014;2014:305784.

27. Sahin K, Tekin A, Ozdas S, Akın D, Yapislar H, Dilek AR et al. Evaluation of carbapenem resistance using phenotypic and genotypic techniques in Enterobacteriaceae isolates. Ann Clin Microbiol Antimicrob 2015;14:44.

28. Pollett S, Miller S, Hindler J, Uslan D, Carvalho M, Humphries RM. Phenotypic and molecular characteristics of carbapenem-resistant Enterobacteriaceae in a health care system in Los Angeles, California, from 2011 to 2013. J Clin Microbiol 2014;52:4003-9. 